\title{
SECURING MUSIC FOR COPYRIGHT PROTECTION
}

\author{
ShreenidhiShastry K.M. ${ }^{1}$, Nimisha M. Shaju ${ }^{2}$, V. Sreenisha ${ }^{3}$, Richard D'souza ${ }^{4}$ \\ ${ }^{1} 8$ th Sem ECE, T.John Institute of Technology, Gottigere, Bangalore-83. \\ ${ }^{2} 8$ th Sem ECE, T.John Institute of Technology, Gottigere, Bangalore-83. \\ ${ }^{3} 8$ th Sem ECE, T.John Institute of Technology, Gottigere, Bangalore-83. \\ ${ }^{4} 8$ th Sem ECE, T.John Institute of Technology, Gottigere, Bangalore-83
}

\begin{abstract}
In recent times, due to the developments in technology the piracy process is less complicated and more common. There is a need to secure the digital data especially audio and in this paper we focus on methods to reduce piracy as well as unauthorized use of audio. Watermarking technique is one of the methods explained in this paper to overcome the above problem. There are different methods to watermark an audio signal, one such method is Phase Coding. In this method, the watermark signal is embedded in the phase of the host signal. The watermark signal is extracted from the attacked audio signal to prove the ownership or the authenticity of the audio signal.
\end{abstract}

Keywords: Watermark, Phase Coding, Embedding, Extraction.

\section{INTRODUCTION}

Before invention of internet and digital media piracy was very limited. Piracy with respect to audio can be defined as copying or distributing of copies of a piece of music for which the owner did not give consent. 12.5 billion dollars in economic loss each year due to piracy in audio signal; $95 \%$ of music downloaded online is illegal. This can be reduced by the method of watermarking. Watermarking is a technique of hiding digital information in the carrier signal. The watermark signal must be transparent, robust, efficient and independent. Watermark is of two types: Secret watermark and Public watermark. The type of watermark used in audio watermarking is secret watermark, which increases the security of the audio signal. Phase coding exploits the fact that the human auditory system has a low sensitivity against relative phase change. Phase coding is a method in which the watermark is embedded in the phase part of the audio signal. In this proposed method, the host signal is divided into number of frames and the first frame is embedded with the watermark. If the owner of the audio signal becomes aware of the unauthorized third party using his audio, then he can prove his ownership by extracting the embedded watermark.

In the next section we talk about related works (2), block diagram (3), methodology of phase coding (4), future scope (5), conclusion (6) and references.

\section{RELATED WORK}

Bhuvanesh Kumar Singh et.al [1] proposes a Digital audio watermarking method to hide the Watermark data into a digital signal. The watermark signal is very difficult to distinguish from the audio signal. If the signal is copied by a third party, the watermark is also carried with the host signal. In this paper, watermark is used for protecting multimedia data from unauthorized copying, piracy, ownership, authentication etc.

Nhut Minh Ngo et.al [2] proposes an audio watermarking method on phase coding. The technique of quantization index modulation is used to embed watermarks into the phase of audio signal. Most of the audio information is present in low frequencies. Hence he suggests to use this frequency range for embedding the watermark and thereby increasing the robustness of the watermark. Change of phase causes sound distortion in the watermarked audio signal. Therefore, the phase difference can be added to reduce this distortion and this is also shown in the result of this paper.

Aree A. Mohammed et.al [3] proposes a method of phase coding to embed any watermark data type (text, image, and audio) in audio signals. In this paper the method is to segment the audio signal and use the Discrete Fourier Transformation (DFT) to convert the signal from time domain to frequency domain. The watermark will be in the first frame's phase. Audio files type must be of WAV type. The idea of this method was tested and the results were obtained by means of normalized correlation (NC) and bit error rates (BER) to determine the robustness of the watermarked signal. This method also increases the inaudibility of the watermark.

\section{BLOCK DIAGRAM}

\subsection{Transmitter}

The audio file is sampled at $44.1 \mathrm{KHz}$ which is the standard sampling frequency for Compact Disk (CD) audio file. This is the host signal for this technique. The Phase Coding method requires the audio not to be silent in the beginning of the audio signal. The watermark signal in this technique is abinary data of 16 bits which is known only to the encoder/ owner. Synchronization code is used to find where the watermark signal is located in the host signal. But in 
Phase coding method this has no significance as the watermark is encoded only in the beginning of the audio signal. The synchronization code and the watermarked signal is combined together and is embedded at the beginning of the host signal. This will result in the watermarked signal.

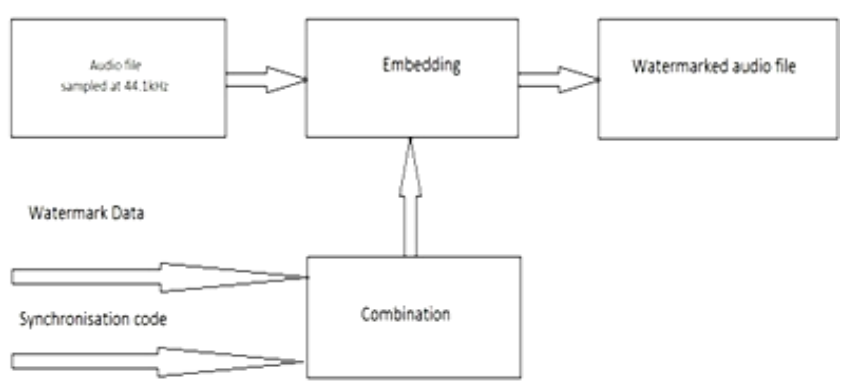

Figure 1: Transmitter

\subsection{Receiver}

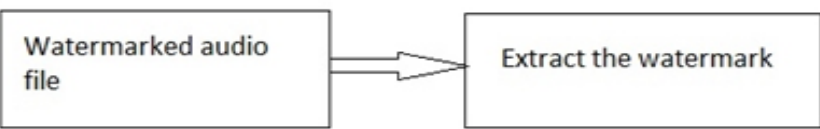

Fig -1: Receiver

The attacked signal is received from the unauthorized third party and is subjected to watermark detection algorithm to extract the watermark signal if available. This extracted watermark phase is compared with the embedded watermark, if it is found to be same then both the signals are one and the same. This will prove the ownership of the audio signal.

\section{ALGORITHM}

\subsection{Watermark Embedding}

- Let $\mathrm{S} 0$ be the host signal where $\mathrm{n}=1<=\mathrm{n}<=\mathrm{N} 0$ where NO is the maximum of $n$

- This S0 is split into frames (gi) with the maximum number of frames being NP. These frames will have $\mathrm{N}$ samples.

- $\mathrm{Np}$ is calculated using the formula $\mathrm{Np}=\mathrm{N} 0 / \mathrm{N}$

- Initialize the watermarked signal as SW

- S0 (n) is initialized with the frames which has a total number of frames as NP.

- Binary watermarked signal WO $(\mathrm{m})$ is a binary random number with the number bits as N2-N. This will usually be of 16 bits.

- Loop through every frame from 1 to NP and calculate amplitude and phase using fast Fourier transform (FFT) algorithm.

- Now, select only the first frame and embed the watermark into this frame.

- This is carried out using a loop with the length 1 to N2-1 and check watermark signal W0 $(\mathrm{m})=1$, if so then change the phase to pi/2 else if W0 (m) change the phase to -pi/2.

- This change in phase for the first frame has to be synchronized so we calculate the phase difference between the old phase and new phase of the first frame.
- This phase difference is added to rest of the frames, by this way we achieve synchronization.

- Using inverse fast Fourier transform (IFFT), we reconstruct the watermarked signal. This will also convert SW i.e. watermarked signal from frequency domain to time domain.

\subsection{Watermark extraction}

- Let SA be the attacked signal. Using FFT, convert this signal into frequency domain with magnitude and phase.

- Select only the phase spectrum and if the phase is greater than or equal to 1 then the extracted watermarked bit will be 1 , else if phase is less than 0 , then the watermark bit will be 0 .

- This extracted watermarked signal is compared with the embedded watermark signal and the respective bit error ratio (BER) is calculated.

- If $B E R=0.00$ that means that the attacked signal is same as the original signal.

- Based on this BER value the ownership of the audio signal can be proved.

\section{Experimental result}

The above algorithm was used in Matlab and watermark technique using phase coding was successfully implemented.

The BER (bit error rate) for the above algorithm was found to be $0.00 \%$. This indicates that the attacked signal had the same watermark that was embedded in the host signal. Suppose if the attacked signal was not that of the owner then the BER value would be more than $50 \%$.

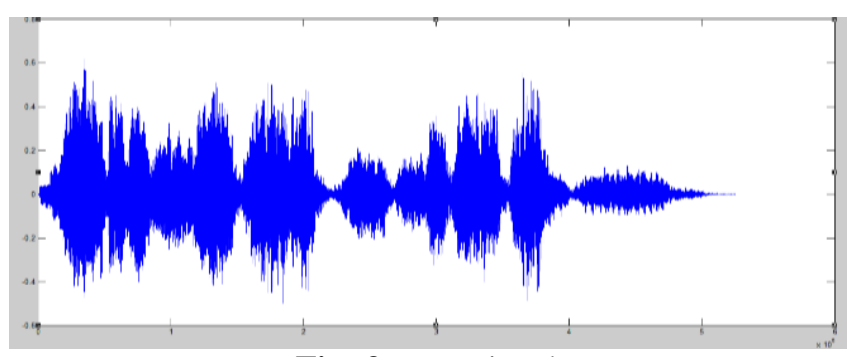

Fig -3: Host signal

- The above graph represents the host signal before watermark.

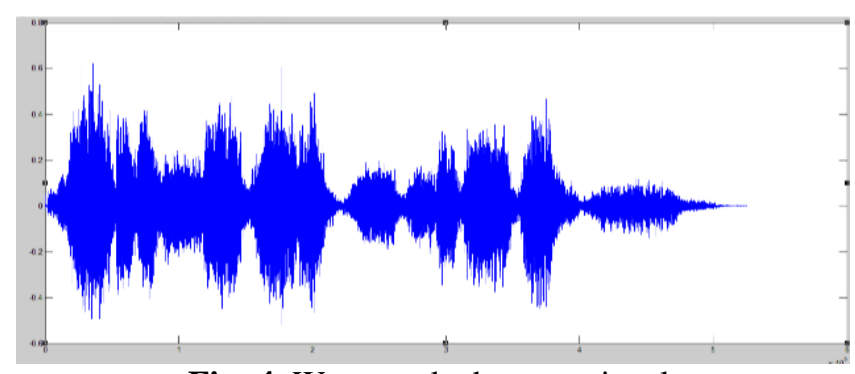

Fig -4: Watermarked output signal

- The above figure represents the graph of Watermarked signal by using the phase coding method. 


\section{FUTURE SCOPE}

This watermarking method can be applied to live performances, thereby avoiding the theft of the live performing music of the respective musicians. This method can also be used for authenticating the audio part in the videos.

\section{CONCLUSION}

This paper has an algorithm that can be used for watermarking the audio file. Thus the problem of authentication of music is possible. This will benefit many young and amateur music composers to prove their ownership.Due to the sudden change in the phase introduces an audible distortion in the watermarked signal. Since the watermark is present only in the first frame of the signal, it can be removed

\section{REFERENCES}

[1]. Bhuvnesh Kumar Singh (M.Tech.), Alok Kumar Singh (Asstt. Prof.) Digital Audio Watermarking: An Overview, International Journal of Electronics and Computer Science Engineering pg. no.1231-1235. ISSN- 2277-1956.

[2]. Nhut Minh Ngo, Brian Michael Kurkoski, and Masashi Unoki, Robust and reliable audio watermarking based on Dynamic phase coding and error control coding, 23rd European Signal Processing Conference (EUSIPCO) 2015.

[3]. Aree A. Mohammed, Diman M. Mohammed, Modified Phase Coding Audio Watermarking Resistant to Signal Attack, International Journal of Computer Applications (0975 - 8887) Volume 92 No.2, April 2014.

[4]. Yiqing Lin, Waleed H. Abdulla, Audio Watermark, A Comprehensive Foundation Using Matlab, Springer

[5]. M. Narimannejad and S. M.Ahadi, "Watermarking of speech signal through phase quantization of sinusoidal model," in Proc. of Iranian Conf. on Elec. Engineering 2011, pp. 1-4.

[6]. N. Khademi, M. A. Akhaee, S. M. Ahadi, M. Moradi, and A. Kashi, "Audio watermarking based on quantization index modulation in the frequency domain," in Proc. of Int. Conf. on Signal Proc. and Comm., pp. 1127-1130, 2007.

[7]. L. Gang, A. N. Akansu, and M. Ramkumar, "MP3 resistant oblivious steganography," in ICASSP 2001, pp. 1365-1368.

[8]. N. M. Ngo and M. Unoki, "Robust and reliable audio watermarking based on phase coding," in ICASSP 2015, pp. 345-349.

\section{BIOGRAPHIES}

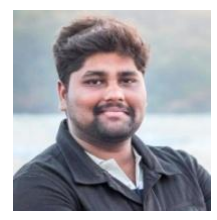

\section{Shreenidhi Shastry K.M}

(shrinidhishastry@gmail.com)

$8^{\text {th }}$ sem, ECE, TJIT.

Have good knowledge in Matlab, C,C++,

VLSI.

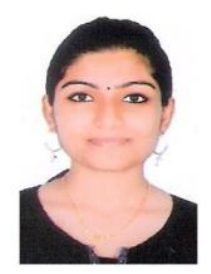

Nimisha M Shaju

(nimishashaju94@gmail.com)

$8^{\text {th }}$ sem, ECE, TJIT.

Have good knowledge in $\mathrm{C}, \mathrm{C}++$ and JAVA.

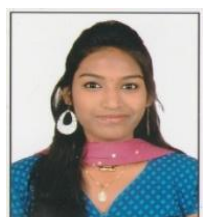

\section{Sreenisha}

(sreeshanair11@gmail.com) $8^{\text {th }}$ sem, ECE, TJIT.

Have good knowlwge in Networking, operating systems

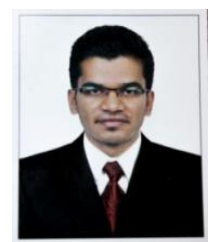

\section{Richard D'souza}

(richarddsouza93@gmail.com)

$8^{\text {th }}$ sem, ECE, TJIT.

Have good knowledge in Networking, control system, $\mathrm{C}, \mathrm{C}++$. 